\section{Genotypic Variation in and Environmental Variance Components of Sugar Composition in Japanese Pear Fruit}

\author{
Toshihiro Saito ${ }^{1}$, Norio Takada, Hidenori Kato, Shingo Terakami, \\ and Sogo Nishio \\ Institute of Fruit Tree and Tea Science, NARO, 2-1 Fujimoto, Tsukuba, \\ Ibaraki 305-8605, Japan
}

Additional index words. broad-sense heritability, fruit sample size, genetic variance, Pyrus pyrifolia, yearly repetition

\begin{abstract}
Genotypic variations in and environmental variance components of the total sugar content (TSC) and sugar composition, including sucrose (SUC), fructose (FRU), glucose (GLU), and sorbitol (SOR), in the fruit juice of 13 Japanese pear cultivars were analyzed. The TSC of 'Kanta' and TSC of 'Hoshiakari' were high (both $>14.5 \mathrm{~g} / 100 \mathrm{~mL})$. The contents of SUC and FRU were higher than those of the other sugars. The SUC contents were ranked as follows: 'Gold Nijisseiki', $7.3 \mathrm{~g} / 100 \mathrm{~mL}$; 'Shuurei', $6.2 \mathrm{~g} / 100 \mathrm{~mL}$; and 'Akizuki', 6.1 g/100 mL. The FRU content in 'Kanta' was the highest among all monomeric sugars evaluated $(6.8 \mathrm{~g} / 100 \mathrm{~mL})$. These results suggest that 'Kanta' is superior in terms of both TSC and sugar composition, which determine sweetness. The yearly environmental variance components were negligible for all traits. The genotype $\times$ year ranged from $4.4 \%$ to $13.7 \%$ of the total variance. Within-tree variance was $17.1 \%$ for TSC, whereas that for the sugar composition ranged from $1.4 \%$ to $6.1 \%$. The tree $\times$ year ranged from $\mathbf{2 . 7 \%}$ to $\mathbf{7 . 4 \%}$. Variance among fruits within trees was the largest environmental variance component-except for FRU—and ranged from $8.8 \%$ to $35.6 \%$. Broad-sense heritability $\left(h_{B}^{2}\right)$ values based on single tree, single year, and single fruit measurements were $0.33,0.64,0.69,0.71$, and 0.76 for TSC, SUC, FRU, GLU, and SOR, respectively. These results suggest that it would be easier to estimate genetic differences in sugar components with a higher level of precision than those in TSC. Increasing the fruit number up to five, in combination with yearly repetition increased to two (without tree repetition), significantly increased the $h_{B}{ }^{2}$ of all traits undergoing study. The information obtained during this study will be useful for improving the accuracy of phenotypic selection and future genomic-based breeding studies performed to improve the sweetness of Japanese pear fruits.
\end{abstract}

Japanese pear (Pyrus pyrifolia Nakai), a member of the genus Pyrus (Rosaceae, Pyrinae) is cultivated throughout Japan. Pear fruit is commercially important, and Japanese pear production is estimated to be more than 70 billion yen per year, which ranks fourth among fruit-tree crops after citrus, apple, and grape production. Pear breeding in Japan started in the early 20th century (Machida, 1979), with fruit quality improvement being the most important objective of breeding programs. Important fruit quality parameters include texture, sweetness, and acidity. Improvements in fruit texture are particularly important because most traditional cultivars present a gritty texture, hence the name "sand pear." 'Kosui', 'Hosui', and 'Akizuki', released in 1959, 1972, and 1998, respectively,

Received for publication 25 Mar. 2019. Accepted for publication 29 May 2019.

We thank Dr. Takeshi Hayashi, Leader of the Breeding Informatics Research Unit, Institute of Crop Science, NARO, for his help discussing the results of this study.

${ }^{1}$ Corresponding author. E-mail: saito@affrc.go.jp. are cultivars with fine fruit texture that are currently leading the Japanese pear market, with $70 \%$ of all Japanese pear cultivating areas in Japan dedicated to their production (Saito, 2016).

Because of the recent consumer demand for superior fruit quality, sweetness has also become an important target when breeding new cultivars (Nishio et al., 2018; Saito, 2016). Generally, sweetness is highly correlated not only with total sugar content (TSC) but also with the sugar component. The TSC of Asian pear fruit reportedly varies according to the cultivar (Abe et al., 1995; Kajiura et al., 1979a; Moriguchi et al., 1992). Furthermore, significant parent-offspring correlations or regression coefficients were obtained for the soluble solid content (SSC) (Abe et al., 1995, Machida and Kozaki, 1976). These results indicated that genetically high TSC cultivars are desirable as cross-parents for breeding cultivars with high TSC. Mature pear fruit mainly contains the following four sugars: sucrose (SUC), fructose (FRU), glucose (GLU), and sorbitol (SOR) (Kajiura et al., 1979a; Yamaki et al., 1976). These sugars have different levels of sweetness; if SUC is rated as 1, then FRU is rated as 1.50 to 1.75 , GLU is rated as 0.70 to 0.80 , and SOR is rated as 0.55 to 0.70 (Doty, 1976; Pangborn, 1963). In addition, the perception of sweetness of SUC is greater than that of FRU (Kaneshi, 1982). In Rosaceae, SOR is translocated from the sink to source tissues; then, it is converted to FRU and GLU by SOR dehydrogenase (Yamaki and Moriguchi, 1989). SUC is synthesized from SUC 6-phosphate by SUC-phosphate synthase (SPS) and is converted to GLU and FRU by SUC invertase (Yamaki, 2010). In addition, the reversible conversion of SUC and uridine diphosphate to uridine diphosphate-GLU and FRU is catalyzed by SUC synthase. Cultivars of Asian pear also exhibit large variations in sugar composition (Kajiura et al., 1979a; Moriguchi et al., 1992), whereas those of apple (Hecke et al., 2006; Wu et al., 2007) and peach (Byrne et al., 1991; Moriguchi et al., 1990), which also belong to Rosaceae, show fewer variations in sugar composition than that of pear. Therefore, it may be possible to breed highsweetness cultivars with not only high sugar content but also high fructose and sucrose contents.

To efficiently breed cultivars with these traits, molecular markers associated with sugar accumulation and sugar metabolism are needed. Previously, we and our colleagues reported a number of quantitative trait loci (QTL) associated with individual sugar content and TSC by analyzing an $\mathrm{F}_{1}$ population derived from a cross between Japanese pear 'Akizuki' and breeding selection 373-55 (Nishio et al., 2018). Although this information will contribute to breeding pear cultivars with high sweetness, further genetic studies are required to obtain desirable high-sweetness genotypes more efficiently. Therefore, it is important for breeders to obtain precise genetic information from the phenotypic value, because the sugar content and composition are controlled by QTL that respond to environmental conditions such as location, year, tree, and fruit.

An analysis of variance (ANOVA) has been performed to estimate the contribution of genetic and environmental variance components to the determination of fruit traits of sweet cherry (Hansche and Beres, 1966; Hansche and Brooks, 1965), Japanese pear fruit (Kozaki, 1975,1976; Machida and Nishio et al., 2011), Japanese persimmon (Yamada et al., 1993, 2002), grape (Sato et al., 2000), citrus (Hamada et al., 2016; Nonaka et al., 2012), and chestnut (Nishio et al., 2014). These estimates provide information regarding the optimal yearly repetition, tree or vine replication, and number of fruits.

The objectives of this study were to evaluate the genotypic differences in TSC and sugar composition among leading commercial cultivars or new promising cultivars that are used as cross-parents for Japanese pear breeding in Japan and to obtain accurate estimates of environmental variance components and broad-sense heritability $\left(h_{B}{ }^{2}\right)$ of 
TSC, SUC, FRU, GLU, and SOR to breed Japanese pear cultivars with high sweetness. Such estimates will provide optimum measurement repetitions for phenotypic selection and further genomic analyses to develop molecular markers associated with TSC and sugar composition.

\section{Materials and Methods}

Measurements of fruit sugar composition. This study was conducted using trees of 13 Japanese pear cultivars (Table 1) grown at the NIFTS Experimental Station in Tsukuba, Japan. These cultivars are used as crossparents for new Japanese pear cultivars. Three trees per cultivar, age 8 to 22 years in 2014, were repeatedly measured during 2014 to 2017 .

Thirty-seven trees were trained on a horizontal trellis used for the commercial production of pears in Japan (Tamura, 2006), whereas two trees ('Chikusui' and 'Gold nijisseiki') were freestanding and open center-trained. All trees were pruned annually in winter and treated for pests and diseases according to the commercial standard practice of the region. Fruits were thinned to one fruit per three to four fruit clusters in mid May, which is a level commonly used in commercial practice, and harvested during early August to late October, when the fruit ground color changed to light brown. Five fruits were randomly sampled per tree at the peak of harvest.

Sampled fruits were cut in half at the equator level, and pairs of flesh plugs were scooped out with a knife from opposite ends through the sphere center of the lower (including calyx end) half of the fruit (Kajiura et al., 1979b). The juice was extracted from the flesh plugs and stored at $-80{ }^{\circ} \mathrm{C}$ until analysis. During analysis, the juice was thawed and diluted with distilled water to $10 \%$ of its original concentration, and mannitol was added as an internal standard (at 0.3 $\mathrm{mg} \cdot \mathrm{mL}^{-1}$ final concentration) for measurements of SUC, FRU, GLU, SOR, and TSC. The sample solutions were then filtered using TORAST Disc (GLCTD-MCE1345; Shimadzu, Kyoto, Japan) before the determination of the SUC, FRU, GLU, and SOR contents by high-performance liquid chromatography on a Shodex SUGAR SP0810 column fitted with a SP-G guard column (Showa Denko, Tokyo, Japan) maintained at $80{ }^{\circ} \mathrm{C}$ and eluted with water at a flow rate of 0.8 $\mathrm{mL} \cdot \mathrm{min}^{-1}$.

Statistical analysis. The sugar content and TSC of each fruit were subjected to ANOVA using the following random model:

$$
\begin{aligned}
\text { Pijkl } & =\mu+G_{i}+T_{i j}+Y_{k}+(G Y)_{i k} \\
& +(T Y)_{i j k}+E_{i j k l}
\end{aligned}
$$

where $P_{i j k l}$ is the phenotypic value of the ${ }_{l}$ th fruit of the ${ }_{j}$ th tree of the ${ }_{i}$ th genotype during the ${ }_{k}$ th year; $\mu$ is the overall mean; $G_{i}$, is the effect of the ${ }_{i}$ th genotype; $T_{i j}$ is the effect of the ${ }_{j}$ th tree of the ${ }_{i}$ th genotype; $Y_{k}$ is the effect of the ${ }_{k}$ th year; $G Y_{i k}$ is the interaction between the ${ }_{i}$ th genotype and the ${ }_{k}$ th year; $T Y_{i j k}$ is the interaction between the ${ }_{j}$ th tree of the ${ }_{i}$ th genotype and the ${ }_{k}$ th year; and $E_{i j k l}$ is the residual of the ${ }_{i}$ th fruit of the ${ }_{j}$ th tree of the ${ }_{i}$ th genotype during the ${ }_{k}$ th year. The ANOVA provided estimates of variances among genotypes $\left(\sigma_{\mathrm{g}}{ }^{2}\right)$, among trees within genotype $\left(\sigma_{\mathrm{t}}^{2}\right)$, among years $\left(\sigma_{\mathrm{y}}^{2}\right)$, genotype $\times$ year $\left(\sigma_{\text {gy }}{ }^{2}\right)$, tree $\times$ year $\left(\sigma_{\text {ty }}{ }^{2}\right)$, and among fruits within trees $\left(\sigma_{\mathrm{f}}^{2}\right)$. The distribution of residual estimates approached a normal distribution at $P=0.05$ (not significant) using a one-sample for Kolmogorov-Smirnov test (Campbell, 1974).

The total environmental variance $\left(\sigma_{\mathrm{E}}^{2}\right)$ was calculated as:

$$
\begin{aligned}
\sigma_{\mathrm{E}}^{2}= & \sigma_{\mathrm{y}}^{2} / y+\sigma_{\mathrm{gy}}^{2} / y+\sigma_{\mathrm{t}}^{2} / t \\
& +\sigma_{\mathrm{ty}}^{2} / t y+\sigma_{\mathrm{f}}^{2} / t y f
\end{aligned}
$$

where $y$ is the number of yearly repetitions, $t$ indicates the tree replications per genotype, and $f$ is the number of fruits per tree. We defined heritability $\left(h_{B}^{2}\right)$ as: $h_{B}^{2}=\sigma_{\mathrm{g}}{ }^{2} /$ $\left(\sigma_{\mathrm{g}}^{2}+{\sigma_{\mathrm{E}}}^{2}\right)$

The ANOVA was calculated using SAS JMP v. 11.0.0. software (SAS Institute, Cary, NC).

\section{Results and Discussion}

The mean TSC and sugar compositions among cultivars ranged from 12.3 to $14.6,2.6$ to $7.3,3.3$ to $6.8,0.4$ to 2.4 , and 1.6 to $4.8 \mathrm{~g} /$ $100 \mathrm{~mL}$ for TSC, SUC, FRU, GLU, and SOR, respectively (Table 2). The overall mean values for each sugar were 13.4, 4.7, 4.4, 1.3 , and $3.0 \mathrm{~g} / 100 \mathrm{~mL}$ for TSC, SUC $(\approx 35 \%$ of TSC), FRU ( $33 \%$ of TSC), GLU ( $10 \%$ of TSC), and SOR (23\% of TSC), respectively. Regarding differences among cultivars for each type of sugar content, the TSC of 'Kanta' (14.6 g/100 mL) and TSC of 'Hoshiakari' $(14.5 \mathrm{~g} / 100 \mathrm{~mL})$ were more than $1.0 \mathrm{~g} /$ $100 \mathrm{~mL}$ higher than the overall mean TSC. In addition, the SUC contents of 'Gold Nijisseiki' (7.3 g/100 mL), 'Shuurei' (6.2 g/100 $\mathrm{mL})$, and 'Akizuki' (6.1 g/100 mL) and the FRU content of 'Kanta' ( $6.8 \mathrm{~g} / 100 \mathrm{~mL})$ were more than $1.0 \mathrm{~g} / 100 \mathrm{~mL}$ higher than the overall mean value, respectively. Conversely, the GLU contents of 'Kanta' and 'Gold Nijiseiki' were almost zero. The SOR contents of 'Gold Nijiseiki' and 'Akizuki' were less than $1.0 \mathrm{~g} / 100 \mathrm{~mL}$ of the overall mean. These results indicated that 'Kanta' has an adequate TSC and sugar composition, because both were relatively high in this cultivar. 'Kanta' is a hybrid between 'Oushuu' and 'Akizuki'. Because 'Oushuu' contains a high FRU content and 'Akizuki' contains a high SUC content, 'Kanta' inherited the traits for high FRU and SUC contents from each parent. It is important to elucidate the effectiveness of obtaining genotypes that contain both high SUC and FRU

Table 1. Japanese pear cultivars used in this study and their origin. ${ }^{z}$

\begin{tabular}{ll}
\hline Cultivar & \multicolumn{1}{c}{ Origin } \\
\hline Akizuki & F1 of 162-29 (Niitaka $\times$ Hosui) $\times$ Kosui \\
Chikusui & Hosui $\times$ Hakkou \\
Gold Nijisseiki & Induced mutant of Nijisseiki \\
Hoshiakari & $314-32($ Kinchaku $\times$ Hosui) $\times$ Akiakari \\
Hosui & Kosui $\times$ Hiratsuka 1 (Ishiiwase $\times$ Nijisseiki) \\
Kanta & Oushuu $\times$ Akizuki \\
Kosui & Kikusui $\times$ Wasekouzou \\
Narumi & $162-29 \times 269-21($ Hosui $\times$ Osa Nijisseiki) \\
Natsushizuku & Chikusui $\times$ Hiratsuka 25 (Kosui $\times$ Kikusui) \\
Niitaka & Amanogawa $\times$ Choujuurou \\
Oushuu & C2 (Laiang Ci Li $\times$ Nijisseiki) $\times$ Shinsetsu \\
Rinka & $269-21 \times$ Akiakari \\
Shuurei & Kosui $\times$ Chikusui \\
\hline
\end{tabular}

${ }^{\mathrm{z}}$ All cultivars including origins are Pyrus pyrifolia Nakai except for Layang Ci Li (P. ussuriensis Maxim.).

Table 2. Genotypic differences in the total sugar content and sugar composition among the 13 Japanese pear cultivars.

\begin{tabular}{lccccc}
\hline & \multicolumn{5}{c}{ Content $(\mathrm{g} / 100 \mathrm{~mL})$} \\
\cline { 2 - 6 } Cultivar & TSC & SUC & FRU & GLU & SOR \\
\hline Kanta & 14.6 & 5.1 & 6.8 & 0.4 & 2.3 \\
Hoshiakari & 14.5 & 2.6 & 4.8 & 2.3 & 4.8 \\
Shuurei & 14.1 & 6.2 & 3.8 & 1.2 & 2.9 \\
Chikusui & 13.9 & 3.8 & 3.9 & 1.9 & 4.3 \\
Niitaka & 13.6 & 5.7 & 3.7 & 1.2 & 3.0 \\
Hosui & 13.5 & 4.2 & 4.6 & 1.5 & 3.2 \\
Natsushizuku & 13.4 & 3.5 & 4.6 & 1.5 & 3.8 \\
Kosui & 13.4 & 5.4 & 3.8 & 1.2 & 3.0 \\
Narumi & 13.1 & 2.8 & 4.3 & 2.4 & 3.6 \\
Oushuu & 12.9 & 3.4 & 5.4 & 1.2 & 2.9 \\
Rinka & 12.8 & 5.2 & 3.9 & 0.9 & 2.8 \\
Gold Nijisseiki & 12.6 & 7.3 & 3.3 & 0.4 & 1.6 \\
Akizuki & 12.3 & 6.1 & 3.8 & 0.7 & 1.7 \\
Average & 13.4 & 4.7 & 4.4 & 1.3 & 3.0 \\
\hline TSC & SUC & &
\end{tabular}

$\mathrm{TSC}=$ total sugar content; $\mathrm{SUC}=$ sucrose; FRU = fructose; $\mathrm{GLU}=$ glucose; $\mathrm{SOR}=$ sorbitol. 
contents by crossing these parental cultivars. Further studies are needed to elucidate whether obtaining QTL associated with both high SUC and FRU contents and accumulating them will be effective for breeding Japanese pear cultivars with high sweetness.

We calculated phenotypic correlation coefficients and their significance for all trait combinations (Table 3 ). The TSC was positively correlated with FRU (0.34), GLU (0.21), and SOR (0.52); in contrast, SUC showed strong negative correlations with FRU (-0.51), GLU (-0.78), and SOR $(-0.55)$. On the contrary, FRU correlated positively with GLU (0.14), whereas GLU correlated positively with SOR (0.66).

The ANOVA showed that the effect of genotype was highly significant $(P<0.01)$ (Table 4). Among the environmental variance components, the effect of year was not significant $(P=0.05)$ for any trait, whereas the effect of the genotype $\times$ year was highly significant. The effect of trees within genotypes was highly significant $(P<0.01)$ for TSC and SOR, significant $(P<0.05)$ for FRU and GLU, and not significant for SUC $(P=$ $0.05)$.

For other fruit tree species, the effect of trees within genotypes on the SSC was not significant, similar to citrus (Hamada et al., 2016), grape (Sato et al., 2000), and persimmon (Yamada et al., 1993). These results suggested that it is important to increase the TSC in pear fruit by appropriately controlling the condition of trees. The effect of tree $x$

Table 3. Correlation coefficients calculated for each pair of traits

\begin{tabular}{lclccc}
\hline & TSC & SUC & FRU & GLU & SOR \\
\hline TSC & 1 & 0.03 & $0.34^{* *}$ & $0.21^{* *}$ & $0.52^{* *}$ \\
SUC & & 1 & $-0.51^{* *}$ & $-0.78^{* *}$ & $-0.55^{* *}$ \\
FRU & & & 1 & $0.14^{* *}$ & 0.06 \\
GLU & & & & 1 & $0.66^{* *}$ \\
SOR & & & & & 1 \\
\hline$* * P<0.01$. & & & &
\end{tabular}

year was highly significant $(P<0.01)$ for all traits.

The contribution of variance components to the total variance differed among traits (Table 5). The contribution of genetic variance $\left(\sigma_{\mathrm{g}}{ }^{2}\right)$ for each sugar component was the highest, ranging from $64.0 \%$ to $75.8 \%$ of the total, indicating that it would be easy to estimate genetic differences in these traits with a high level of precision. In contrast, the contribution of $\sigma_{\mathrm{g}}{ }^{2}$ to the TSC was relatively small $(32.5 \%)$, indicating that it would be difficult to estimate genetic differences in this trait. The reason for the small contribution value of $\sigma_{\mathrm{g}}{ }^{2}$ to TSC, despite large values for each sugar component, was the highly negative correlation among sugar components. If the relationships among individual sugar components were mutually independent, then the contribution of $\sigma_{\mathrm{g}}{ }^{2}$ to TSC would be almost equal to those of each sugar component, because genetic and environmental variances for TSC would be the sum of the four sugar components analyzed.

We identified the acid invertase genes (PPAIV3 and PPAIV1) as promising candidates associated with QTL related to each sugar component (Nishio et al., 2018). Acid invertase is a key enzyme in the conversion of SUC to FRU and GLU, and the acid invertase genes likely do not contribute to increasing the genetic value of TSC, although they contribute to the increasing genetic value of the three sugars.

Regarding the environmental variance component, the effect of year $\left(\sigma_{\mathrm{y}}^{2}\right)$ ranged from $0 \%$ to $0.9 \%$, which was negligible. Conversely, genotype $\times$ year $\left(\sigma_{\mathrm{gy}}{ }^{2}\right)$ ranged from $4.4 \%$ to $13.7 \%$. For FRU $(13.7 \%)$, because genotype $\times$ year was apparently larger than the variance components associated with trees, yearly repetition is more efficient than tree replication for reducing environmental variance.

Variance among trees $\left(\sigma_{t}^{2}\right)$ was $17.1 \%$ for TSC, which was relatively larger than the corresponding values for the four sugar components, which ranged from $1.4 \%$ to $6.1 \%$. Tree $\times$ year $\left(\sigma_{\text {ty }}{ }^{2}\right)$ ranged from $2.7 \%$ to $7.4 \%$. These results suggested that increasing the number of trees is not effective for increasing the accuracy of estimating the genetic effects on sugar content-related traits, except for TSC. The variance among fruits within trees $\left(\sigma_{\mathrm{f}}^{2}\right)$ was the largest among the environmental variance components of TSC $(35.6 \%)$, SUC (22.2\%), GLU (17.2\%), and SOR $(8.8 \%)$, second to $\sigma_{\mathrm{gy}}{ }^{2}$ for FRU $(13.0 \%)$. These results indicated that the TSC and SUC contents would fluctuate more than the other sugar components among fruits from the same tree. This is probably due to differences in fruit development and maturation stage, because the TSC and SUC contents increase rapidly near and during the harvest period of Japanese pear (Moriguchi et al., 1992; Tanase and Yamaki, 2000).

The $h_{B}{ }^{2}$ was calculated to estimate optimal repetition, which enables the evaluation of TSC and each sugar component efficiently with regard to year, tree, and fruit within a tree (Fig. 1). The $h_{B}^{2}$ values were 0.33 for TSC, 0.64 for SUC, 0.69 for FRU, 0.71 for GLU, and 0.76 for SOR during the evaluation using single tree, single year, and single fruit measurements. Only narrowsense heritability for SSC has been previously reported for heritability estimates of the sweetness of Japanese pear fruits [0.50-0.56 (Machida and Kozaki, 1976) and 0.42 (Abe et al., 1995)]. The $h_{B}{ }^{2}$ values of single tree, single year, single fruit measurements for SSC of other fruit trees have been reported as follows: 0.34 for citrus (Hamada et al., 2016), 0.35 for apple (Kunihisa et al., 2014), 0.40 for grape (Sato et al., 2000), and 0.47 for persimmon (Yamada et al., 1993). Our results for TSC were similar to these values. Increasing the yearly repetition to two and using single fruit measurements increased $h_{B}{ }^{2}$ from 0.33 to 0.44 for TSC, 0.64 to 0.77 for SUC, 0.69

Table 4. Analysis of variance of the total sugar content and sugar composition of fruit juice using 13 genotypes with three trees per genotype for 4 years.

\begin{tabular}{|c|c|c|c|c|c|c|c|}
\hline \multirow{2}{*}{ Source of variation } & \multicolumn{6}{|c|}{ Mean square } & \multirow{2}{*}{ Expected mean squared } \\
\hline & \multicolumn{6}{|c|}{ Total sugar content and sugar composition in fruit juice } & \\
\hline Genotype & 12 & $29.38^{* *}$ & $124.28^{* *}$ & $52.56^{* *}$ & $24.88^{* *}$ & $50.83^{* *}$ & $\sigma_{\mathrm{f}}^{2}+5 \sigma_{\mathrm{ty}}{ }^{2}+15 \sigma_{\mathrm{gy}}{ }^{2}+20 \sigma_{\mathrm{t}}{ }^{2}+60 \sigma_{\mathrm{g}}{ }^{2}$ \\
\hline Genotype $\times$ year & 36 & $2.02 * *$ & $4.99 * *$ & $2.82 * *$ & $0.73 * *$ & $1.05^{* *}$ & $\sigma_{\mathrm{f}}{ }^{2}+5 \sigma_{\mathrm{ty}}{ }^{2}+15 \sigma_{\mathrm{gy}}{ }^{2}$ \\
\hline Trees within genotype & 26 & $4.94 * *$ & $2.10^{\mathrm{NS}}$ & $0.70 *$ & $0.35 *$ & $1.64 * *$ & $\sigma_{\mathrm{f}}^{2}+5 \sigma_{\mathrm{ty}}^{2}+20 \sigma_{\mathrm{t}}^{2}$ \\
\hline Tree $\times$ year & 78 & $0.87^{* *}$ & $1.31 * *$ & $0.36^{* *}$ & $0.17 * *$ & $0.34 * *$ & $\sigma_{\mathrm{f}}^{2}+5 \sigma_{\mathrm{ty}}{ }^{2}$ \\
\hline Fruit within tree & 624 & 0.43 & 0.69 & 0.16 & 0.10 & 0.09 & $\sigma_{f}^{2}$ \\
\hline
\end{tabular}

Table 5. Estimates of variance components and their contribution to the total variance obtained by an analysis of variance of the total sugar content and sugar composition using 13 genotypes, three trees per genotype, for 4 years.

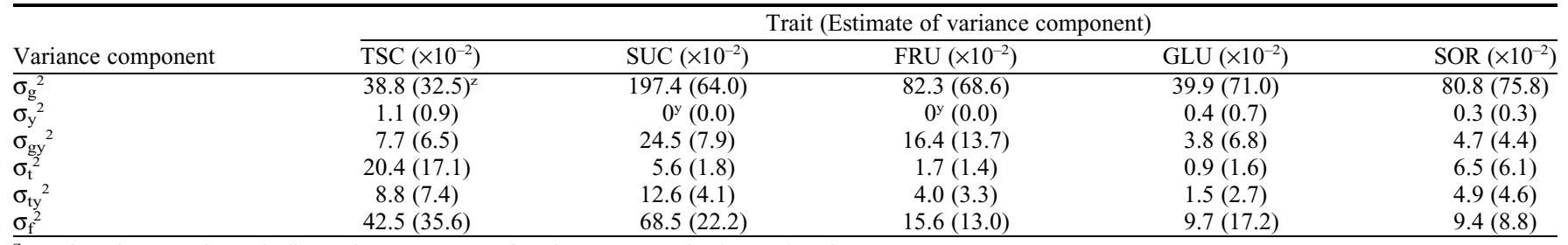

${ }^{\mathrm{z}}$ Numbers in parentheses indicate the percentage of each component in the total variance.

${ }^{\mathrm{y}}$ Negative values were assumed to be zero. 
TSC

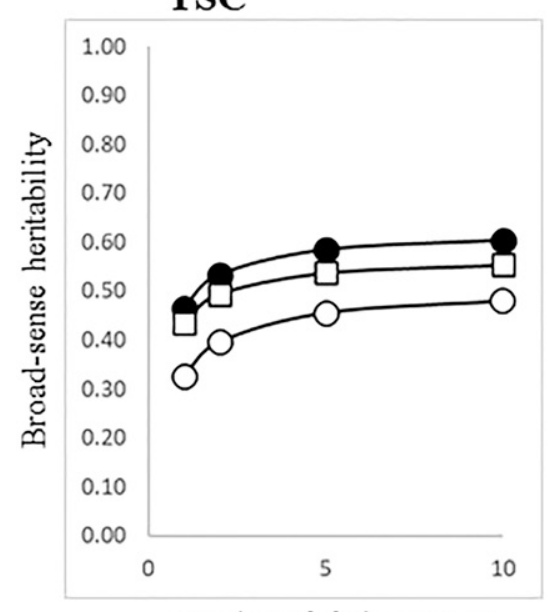

Number of fruits per tree

GLU

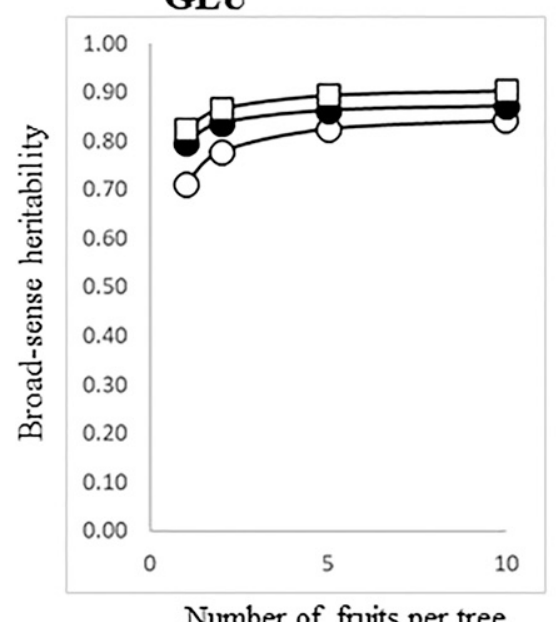

Number of fruits per tree
SUC



Number of fruits per tree

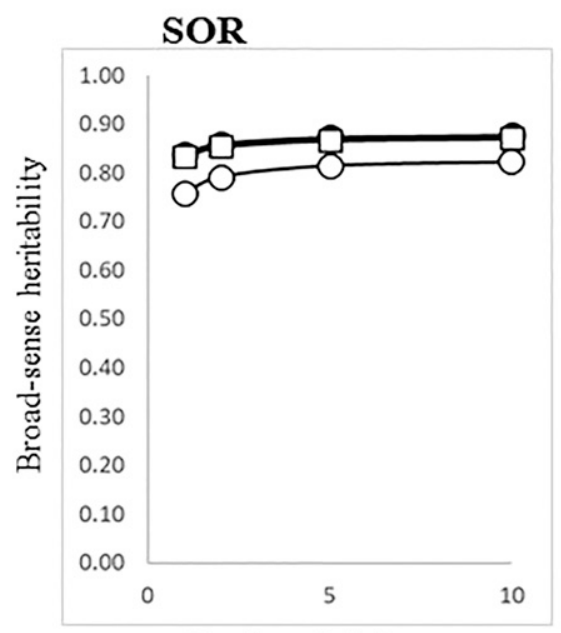

Number of fruits per tree
FRU

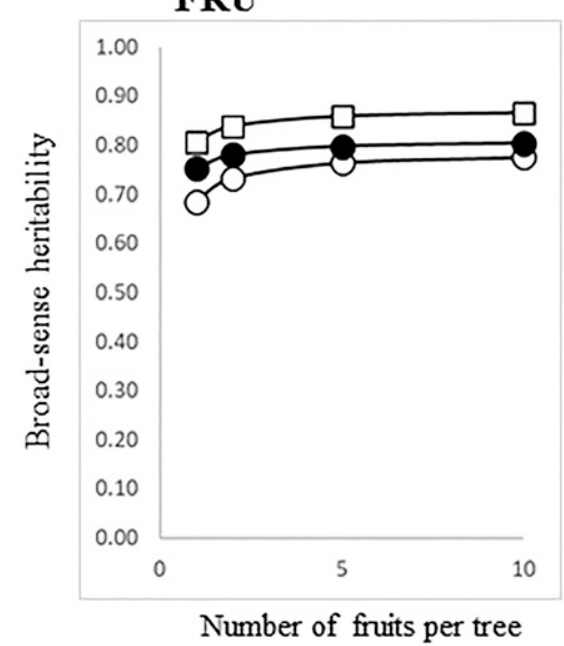

Fig. 1. Effects of the number of fruits per tree, tree replication, and yearly repetition on the broad sense heritability $\left(h_{B}{ }^{2}\right)$ of total sugar content and sugar composition. $\bigcirc=1$ tree and 1 year; $\square=1$ tree and 2 years; $O=2$ trees and 1 year.

to 0.81 for FRU, 0.71 to 0.83 for GLU, and 0.76 to 0.84 for SOR. Increasing the tree number to two trees per genotype increased $h_{B}{ }^{2}$ from 0.33 to 0.47 for TSC, 0.64 to 0.74 for SUC, 0.69 to 0.75 for FRU, 0.71 to 0.80 for GLU, and 0.76 to 0.84 for SOR. These results indicated that the effect of each replication on increasing $h_{B}^{2}$ was almost the same, except for FRU. The reason why the increase in $h_{B}{ }^{2}$ with yearly repetition was slightly higher than with tree replication for FRU was that the environmental variance associated with $\sigma_{\mathrm{gy}}{ }^{2}$, which was the largest environmental variance component for FRU, was not decreased by tree replication, but by year repetition. Increasing the yearly repetition seems more reasonable than increasing the number of tree replications if both increase $h_{B}{ }^{2}$ similarly, because the latter requires more space and effort (Nishio et al., 2014; Sato et al., 2000). Increasing the number of fruits within a tree to five for single year measurements increased $h_{B}^{2}$ from 0.33 to 0.46 for TSC, 0.64 to 0.78 for SUC, 0.69 to 0.76 for FRU, 0.71 to 0.82 for GLU, and 0.76 to 0.82 for SOR. Further increases in the number of fruits within a tree from 5 to 10 for single year measurements resulted in only small increases or no increase in $h_{B}{ }^{2}$ (from 0.46 to 0.48 for TSC, 0.78 to 0.80 for SUC, 0.76 to 0.78 for FRU, 0.82 to 0.84 for GLU, and 0.82 to 0.82 for SOR).

These results indicated that increasing the number of fruits within a tree from one to five was quite effective for accurately evaluating $h_{B}^{2}$ for all traits. However, increasing the number of fruits from 5 to 10 was not so effective. From these results, it was possible to estimate the genetic characteristics of each sugar component by evaluating five fruits per tree per year $\left(h_{B}^{2}, 0.76-0.82\right)$. Moreover, it is desirable to evaluate five fruits per tree with two yearly repetitions for accurate estimation, especially for TSC, SUC, and FRU, which had $h_{B}^{2}$ that increased from 0.46 to $0.54,0.78$ to 0.86 , and 0.76 to 0.86 , respectively; however, yearly repetition is expensive and difficult.

Our estimates of environmental variance components in this study are useful for evaluating not only the relative efficiency of yearly repetition, tree replication, and number of fruits but also genomic-based breeding approaches, including QTL analyses, genome-wide association studies, and genomic selection, all of which require accurate phenotype evaluations and datasets. QTL associated with TSC or SSC have been identified in pear on linkage groups 2, 4, 5, 6, 8, 10, and 14 (Wu et al., 2014; Yamamoto et al., 2014; Zhang et al., 2013). However, no reliable molecular markers that can be applied in practical pear fruit breeding programs for sugar accumulation have been obtained from these studies. Regarding genome-wide association studies and genomic selection for sugar accumulation in pears, the accuracy value of genomewide prediction was low $(<0.20)$ for SSC using phenotypic values characterized as ordinal categorical scores (Iwata et al., 2013). In a subsequent study, the level of accuracy of genomic prediction for genomic selection for SSC was improved to medium $(0.5<r<0.7)$ using phenotypic values characterized as continuous data with large datasets (Minamikawa et al., 2018). These results might be influenced by the low heritability of TSC. However, we identified two QTL associated with the metabolism of 
SUC to hexose on linkage groups 1 and 7, and one QTL associated with sugar accumulation (TSC) on LG 11 from a breeding line during an evaluation using one tree and four fruits per tree with two yearly repetitive measurements (Nishio et al., 2018). On the contrary, sugar components are possibly controlled by dominant effects. Usually, the QTL with additive effects are effectively used for marker-assisted selection. Regarding the QTL with dominant effects, which are caused by allelic interactions in QTL, they can be used effectively for marker-assisted selection if the dominant effects are not significant. When using QTL with large dominant effects, including those with overly dominant effects, for marker-assisted selection, it is possibly insufficient to introduce a single allele of the QTL, but the allele combination of QTL, which causes an overly dominant effect, should be considered and introduced. Although the information will accelerate research and breeding for the improvement of fruit sweetness, further studies are needed to verify the validity of using these QTL in other populations and to identify other QTL that control each sugar component for breeding cultivars with high sweetness. Accurate evaluation of the precise sugar composition and TSC based on sufficient repetition to reduce environmental variance will improve future genomic-based breeding studies performed to improve the sweetness of Japanese pear fruits.

\section{Literature Cited}

Abe, K., Y. Sato, T. Saito, A. Kurihara, and K. Kotobuki. 1995. Narrow sense heritability of fruit characters in Japanese pear (Pyrus pyrifolia Nakai). Breed. Sci. 45:1-5.

Byrne, D.H., A.N. Nikolic, and E.E. Burns. 1991. Variability in sugars, acids, firmness, and color characteristics of 12 peach genotypes. J. Amer. Soc. Hort. Sci. 116:1004-1006.

Campbell, R.C. 1974. Statistics for biologists. 2nd ed. Cambridge Univ. Press, U.K.

Doty, T. 1976. Fructose sweetness: A new dimension. Cereal Foods World 21:62-63.

Hamada, H., K. Nonaka, T. Yoshioka, and M. Yamada. 2016. Environmental variances of fruit traits in a mandarin-related population used in citrus breeding in Japan. HortScience 51:680-683.

Hansche, P.E. and V. Beres. 1966. An analysis of environmental variability in sweet cherry (Prunus avium L.). Proc. Amer. Soc. Hort. Sci. 88:173-183.

Hansche, P.E. and R.M. Brooks. 1965. Temporal and spatial repeatability of a series of quantitative characters in sweet cherry (Prunus avium L.). Proc. Amer. Soc. Hort. Sci. 86:120-128.

Hecke, K., K. Herbinger, R. Veberic, M. Trobec, H. Toplak, F. Stampar, H. Keppel, and D. Grill. 2006. Sugar-, acid- and phenol contents in apple cultivars from organic and integrated fruit cultivation. Eur. J. Clin. Nutr. 60:11361140.

Iwata, H., T. Hayashi, S. Terakami, N. Takada, Y. Sawamura, and T. Yamamoto. 2013. Potential assessment of genome-wide association study and genomic selection in Japanese pear Pyrus pyrifolia. Breed. Sci. 63:125-140.

Kajiura, I., S. Yamaki, M. Omura, T. Akihama, and Y. Machida. 1979a. Improvement of sugar content and composition in fruits, and classifications of east Asian pears by the principal component analysis of sugar compositions in fruits. Jpn. J. Breed. 29:1-12. [In Japanese with English summary].

Kajiura, I., Y. Sato, M. Omura, and Y. Machida 1979b. Local difference in the concentration of soluble solids with a fruit of Japanese pear and the sampling method for the measuring. Bul. Fruit Tree Res. Stn. 6:1-14. [In Japanese with English summary].

Kaneshi, T. 1982. Trends of sweeteners for soft drink. J. Food Sci. 21(2):29-32. [In Japanese].

Kunihisa, M., S. Moriya, K. Abe, K. Okada, T. Haji, T. Hayashi, H. Kim, C. Nishitani, S. Terakami, and T. Yamamoto. 2014. Identification of QTLs for fruit quality traits in Japanese apples: QTLs for early ripening are tightly related to preharvest fruit drop. Breed. Sci. 64:240-251.

Machida, Y. 1979. Trend of breeding works for Japanese pear in Japan. Jpn. Agr. Res. Q. 13:234-237.

Machida, Y. and I. Kozaki. 1975. Quantitative studies on the fruit quality for Japanese pear (Pyrus serotina Rehder) breeding I. Statistical analyses of cultivar populations. J. Jpn. Soc. Hort. Sci. 44:235-240. [In Japanese with English summary].

Machida, Y. and I. Kozaki. 1976. Quantitative studies on the fruit quality for Japanese pear (Pyrus serotina Rehder) breeding II. Statistical analyses of a hybrid seedling population. J. Jpn Soc. Hort. Sci. 44:325-329. [In Japanese with English summary].

Minamikawa, F.M., N. Takada, S. Terakami, T. Saito, A. Onogi, H. Kajiya-Kanegae, T. Hayashi, T. Yamamoto, and H. Iwata. 2018. Genome-wide association study and genomic prediction using parental and breeding populations of Japanese pear (Pyrus pyrifolia Nakai). Sci. Rpt. 8:11994.

Moriguchi, T., Y. Ishizawa, and T. Sanada. 1990. Differences in sugar composition in Prunus persica fruit and the classification by the principal component analysis. J. Jpn. Soc. Hort. Sci. 59:307-312.

Moriguchi, T., K. Abe, T. Sanada, and S. Yamaki. 1992. Levels and role of sucrose synthase, sucrose-phosphate synthase, and acid invertase in sucrose accumulation in fruit of Asian pear. J. Amer. Soc. Hort. Sci. 117:274-278.

Nishio, S., T. Saito, S. Terakami, N. Takada, H. Kato, A. Itai, and T. Yamamoto. 2018. Identification of QTLs associated with conversion of sucrose to hexose in mature fruit of Japanese pear. Plant Mol. Biol. Rpt. 36:643-652.

Nishio, S., M. Yamada, Y. Sawamura, N. Takada, and T. Saito. 2011. Environmental variance components of fruit ripening date as used in both phenotypic and marker-assisted selection in Japanese pear breeding. HortScience 46:1540-1544.

Nishio, S., M. Yamada, N. Takada, H. Kato, N. Onoue, Y. Sawamura, and T. Saito. 2014 Environmental variance and broad-sense heritability of nut traits in Japanese chestnut breeding. HortScience 49:696-700.
Nonaka, K., M. Kita, Y. Ikoma, H. Fukamachi, A. Imai, T. Yoshioka, and M. Yamada. 2012. Genetic differences and environmental variations in carotenoid concentrations of fruit flesh in parental population used in citrus breeding in Japan. J. Amer. Soc. Hort. Sci. 137:243-249.

Pangborn, R. 1963. Relative taste intensities of selected sugars and organic acids. J. Food Sci. 28:726-733.

Saito, T. 2016. Advances in Japanese pear breeding in Japan. Breed. Sci. 66:46-59.

Sato, A., M. Yamada, H. Iwanami, and N. Hirakawa. 2000. Optimal spatial and temporal measurement repetition for reducing environmental variation of berry traits in grape breeding. Scientia Hort. 85:75-83.

Tamura, F. 2006. Japanese pear, p. 50-58. In: Jpn. Soc. Hort. Sci. (ed). Horticulture in Japan. Shoukadoh Publication, Kyoto.

Tanase, K. and S. Yamaki. 2000. Sucrose synthase isozymes related to sucrose accumulation during fruit development of Japanese pear (Pyrus pyrifolia Nakai). J. Jpn. Soc. Hort. Sci. 69:671676.

Wu, J., H. Gao, L. Zhao, X. Liao, F. Chen, Z. Wang, and X. Hu. 2007. Chemical compositional characterization of some apple cultivars. Food Chem. 103:88-93.

Wu, J., L.T. Li, M. Li, M.A. Khan, X.G. Li, H. Chen, H. Yin, and S.L. Zhang. 2014. Highdensity genetic linkage map construction and identification of fruit-related QTLs in pear using SNP and SSR markers. J. Expt. Bot. 65:5771-5781.

Yamada, M., H. Yamane, K. Yoshinaga, and Y. Ukai. 1993. Optimal temporal and spatial measurement repetition for selection in Japanese breeding. HortScience 28:838841.

Yamada, M., A. Sato, and Y. Ukai. 2002. Genetic differences and environmental variations in calyx-end fruit cracking among Japanese persimmon cultivars and selections. HortScience 37:164-167.

Yamaki, S. 2010. Metabolism and accumulation of sugars translocated to fruit and their regulation. J. Jpn. Soc. Hort. Sci. 79:1-15.

Yamaki, S., I. Kajiura, M. Omura, and K. Matsuda. 1976. Watercore in Japanese pear (Pyrus serotina Rehder var. 'Culta' Rehder). II. Chemical changes in watercored tissue. Scientia Hort. 4:271-277.

Yamaki, S. and T. Moriguchi. 1989. Seasonal fluctuation of sorbitol-related enzymes and invertase activities accompanying maturation of Japanese pear (Pyrus serotina Rehder var. culta Rehder) fruit. J. Jpn. Soc. Hort. Sci. 57:602607.

Yamamoto, T., S. Terakami, N. Takada, S. Nishio, N. Onoue, C. Nishitani, M. Kunihisa, E. Inoue, H. Iwata, T. Hayashi, A. Itai, and T. Saito. 2014. Identification of QTLs controlling harvest time and fruit skin color in Japanese pear (Pyrus pyrifolia Nakai). Breed. Sci. 64:351361.

Zhang, R., J. Wu, X. Li, M.A. Khan, H. Chen, S.S Korban, and S. Zhang. 2013. An AFLP, SRAP, and SSR genetic linkage map and identification of QTLs for fruit traits in pear (Pyrus L.). Plant Mol. Biol. Rpt. 31:678-687. 